\title{
Semi-automatic system for non-invasive diagnosis of bronchial asthma based on microwave technologies
}

\author{
Ivan V. Semernik ${ }^{*}$, Alexander $V$. Dem'yanenko, and Feruz S. Topalov \\ Southern Federal University, Department of Antennas and Radio Transmitting Equipment, 347922 \\ Nekrasovskiy lane 44, Taganrog, Russia
}

\begin{abstract}
In this paper, the structure of a semi-automatic non-invasive harmless diagnostic system for bronchial asthma and other bronchopulmonary diseases are discussed. The components of the diagnostic system are discussed. It is shown that it is possible to design a diagnostic system in a portable compact design by using a laser displacement sensor instead of a bulky positioning system. The advantages of the proposed system are the simplicity of the implementation of the diagnostic system and examination, harmlessness, non-invasiveness of the examination procedure, no need to perform breathing maneuvers. These advantages allow us to consider the proposed system as an addition to the existing methods of diagnosing bronchial asthma, as well as an express method for diagnosing bronchopulmonary diseases or a system for periodically monitoring the health of a patient undergoing inpatient treatment or not having permanent access to a large medical center equipped with expensive equipment.
\end{abstract}

\section{Introduction}

According to statistical studies all over the world, more than 300 million people have been diagnosed with bronchial asthma [1]. In some countries of the world, about $20 \%$ of the population suffer from bronchial asthma of varying severity. In addition, the big problem is the increasing cases of the disease in young children. In connection with the foregoing, the development of new methods and devices for the diagnosis and treatment of bronchial asthma, including in young children, is a very urgent task.

A large number of publications, the area of interest of which is the use of modern technologies for the diagnosis, treatment and control of a wide range of various diseases [2$4]$, including bronchial asthma [5,6], indicates the relevance and prospects of this area of research. The use of modern technologies can not only facilitate and accelerate the correct diagnosis, but also expand the scope of accessibility of high-quality medical services in sparsely populated and remote areas.

This paper presents the structure of a semi-automatic system of non-invasive harmless diagnosis of bronchial asthma, based on a radio-frequency scanning of the patient's chest with a microwave probing harmonic signal of a given frequency and subsequent analysis of

*Corresponding author: ivsemernik@sfedu.ru 
the distribution of the microwave signal transmission coefficient. The presented system is harmless, non-invasive and does not require the patient to perform respiratory maneuvers; therefore, it can be used to examine patients of all age groups, including young children.

\section{Diagnostic system structure}

In [7], the authors presented for discussion an automated diagnostic system for bronchopulmonary diseases based on radio frequency scanning of the patient's chest with a microwave probe harmonic signal of a given frequency and subsequent analysis of the distribution of the microwave signal transmission coefficient. The presented system requires further refinement and improvement of characteristics, but from an analysis of the experimental results obtained it can be seen that the system is able to detect heterogeneities (sputum accumulations in the bronchi of medium and small diameter) in the patient's chest, and also to form a map of the heterogeneities distribution for diagnosis and prescription treatment.

For the implementation of radiofrequency scanning of the chest in the automatic diagnostic system, a radiating module positioning system based on the use of stepper motors was used. This system has a high position accuracy. However, in some cases (for example, when used in an ambulance), compactness of equipment is more important. In this regard, this paper presents a semi-automatic diagnostic system that does not require the use of a bulky positioning system of the radiating module. This allows you to significantly reduce the dimensions of the device, and reduce its cost.

The block diagram of the semi-automatic diagnostic system is shown in simplified form in Figure 1.

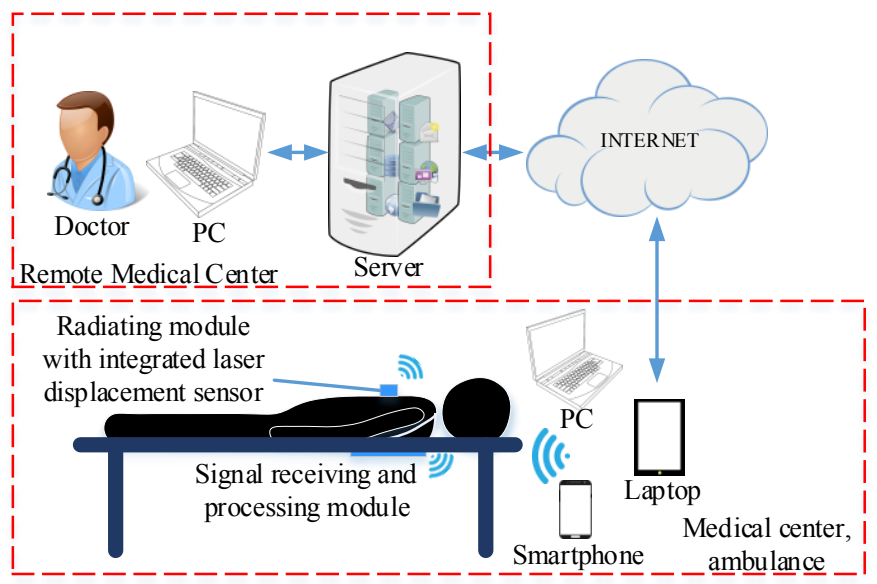

Fig. 1. The block diagram of the semi-automatic diagnostic system.

The main components of the proposed system are the radiating module, which forms the probing microwave signal of a given frequency and radiates it with the help of the antennaapplicator, as well as the signal receiving and processing module, which detects the probing microwave signal and processes it [7].

The main difference between the semi-automatic system of non-invasive diagnosis of bronchopulmonary diseases and the one presented in the work [7] is that in the first one there is no bulky system of automatic positioning of the radiating module. Instead, a laser displacement sensor is integrated into the radiating antenna applicator, which allows tracking the movement of the radiating module in the process of radio frequency scanning relative to the initial point on the patient's chest surface. The accuracy of such a positioning 
system is less, however, for the purposes of radiofrequency scanning of the chest, it is sufficient [8]. The operator along any path that allows covering the entire surface of the patient's chest carries out the movement of the radiating module at the same time manually (eg, meander). In addition, to ensure ease of conducting an examination in conditions of a limited ambulance space or at home, the patient's radiating module and signal receiving and processing module can be made wireless, and the processing and visualization of measurement results can be performed on a PC, tablet or smartphone.

Due to the simpler and more compact positioning system of the radiating module, the semi-automatic non-invasive diagnostics system has smaller dimensions compared to the automated system and significantly lower cost. This allows expanding the spread of such a semi-automatic diagnostic system for bronchopulmonary diseases in small medical centers, as well as equipping an ambulance with a similar system.

As for the automated system, if necessary, the results of the examination can be transferred to the server of a remote large medical center for additional consultation and analysis.

\section{Radiating module}

The block diagram of the radiating module is shown in Figure 2.

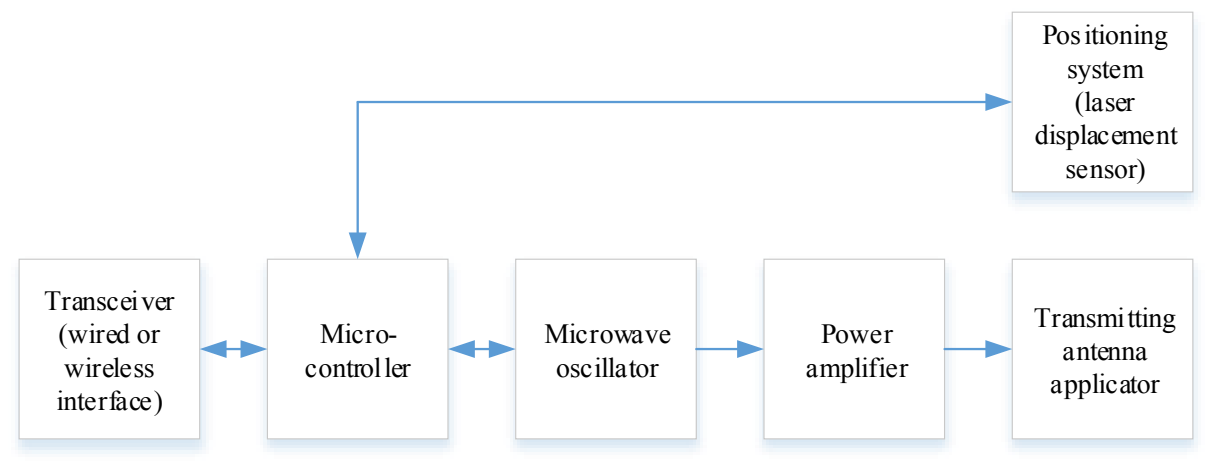

Fig. 2. The block diagram of the radiating module.

The main element of the radiating module, which generates the probing microwave signal of a given frequency, is the ADF4350 frequency synthesizer. The power of the probing signal is controlled using an attenuator integrated into the frequency synthesizer, as well as an ADL5545 chip amplifier installed in the microwave path.

To suppress the parasitic harmonics of the frequency of the probing signal, a band pass filter is used, made on the basis of microstrip technology and tuned to the range of possible frequencies of the probing signal.

The frequency of the probing microwave signal is controlled using an ATMega32 microcontroller. The communication of the radiating module with the PC is carried out via the USB interface. The power of the radiating module is provided from the USB port of the $\mathrm{PC}$, or from the built-in battery in the case of a wireless version of the radiating module.

The output power level of the radiating module does not exceed the established permissible norms [9], which ensure the safety of using the diagnostic system for continuous monitoring.

For tracking the position of the radiating module in the scanning process, the ADNS9500 laser displacement sensor is used, which is installed on a separate PCB and integrated into the transmitting antenna applicator. The sensor positioning and data acquisition is performed by the microcontroller of the radiating module via the SPI interface. 
The appearance of the radiating module with the antenna-applicator installed in the holder made of Plexiglas, as well as with a laser displacement sensor is shown in Figure 3.

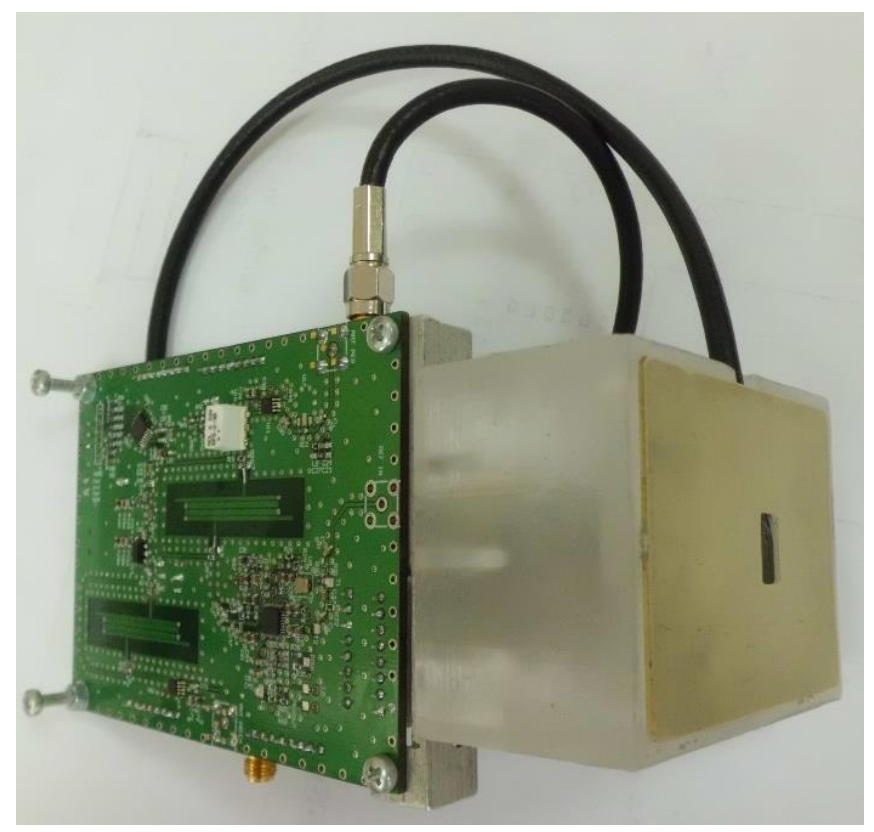

Fig. 3. The appearance of the radiating module.

\section{Experimental results}

In the experimental study, control of the probing signal frequency, collection of the measurements results, collecting of the points coordinates at which measurements were performed, and the post-processing and visualization of the results were carried out using MATLAB. The radiating module and the signal receiving and processing module were connected to the USB ports of the PC, and the data were transferred to MATLAB through the created virtual COM ports.

The probing microwave signal was received by a single receiving antenna applicator located on the back surface of the chest phantom.

Due to the specifics of the laser displacement sensor operation with an uneven underlying surface, as well as in the case of the sensor rotation relative to the direction of movement, an additional erroneous displacement of the position sensor coordinates can be observed, which can be eliminated at the post-processing of measurement results if there is a limiter in one of the scanning directions, which coordinate is taken to be zero.

The trajectory of the radiating module in the case of manual movement and determination of coordinates using a laser displacement sensor is shown in Figure 4,a. Figure 4,b shows the trajectory of the laser displacement sensor during the same scan after applying the correction.

From figure 4,a it can be seen that the accumulation of errors in estimating coordinates during the scanning process results in significant errors in determining the coordinates of the radiating module (about $5 \mathrm{~cm}$ ). Despite the fact that an emphasis was set at the $\mathrm{x}=0$ border, when scanning was performed, the movement trajectory to the right was observed due to the influence of the underlying surface, as well as involuntary rotations of the radiating module to the operation of the laser displacement sensor. The indicated error offset was different for different scans. 

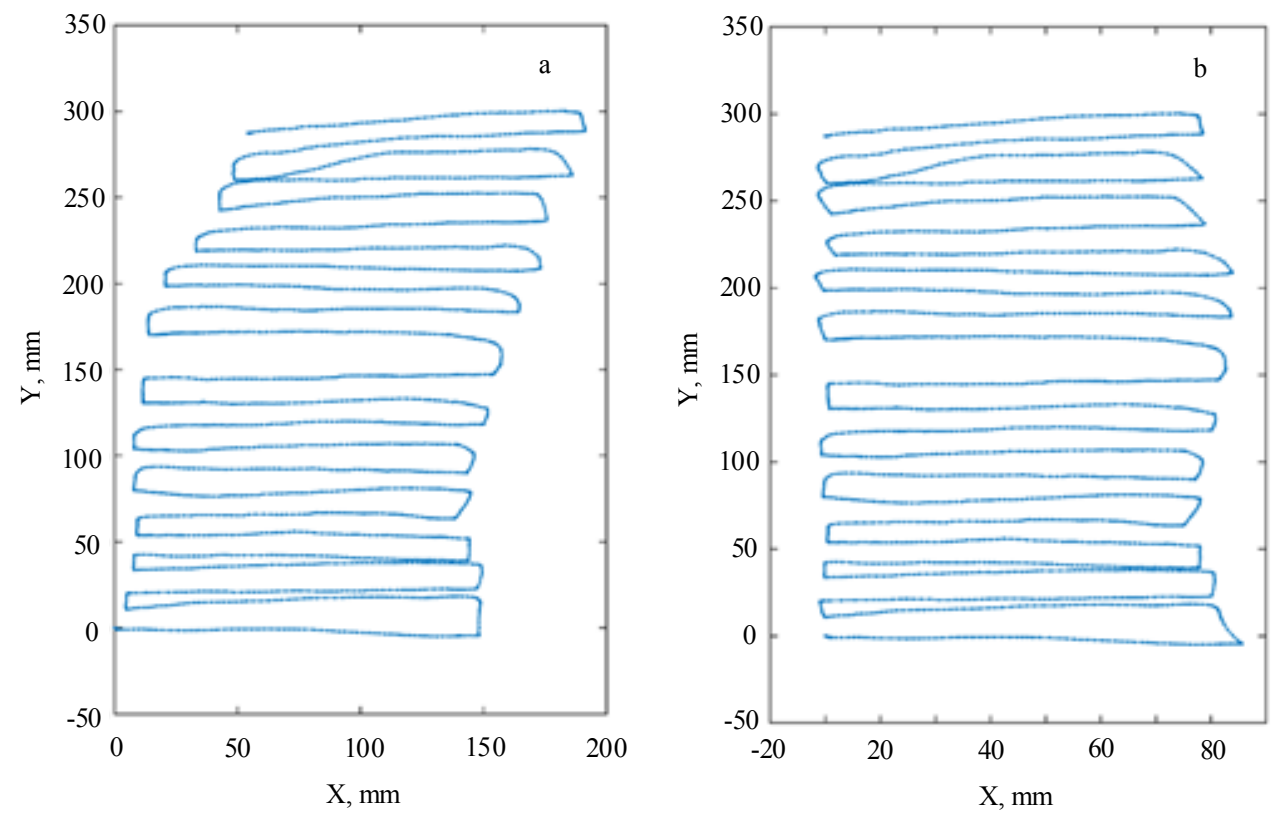

Fig. 4. The trajectory of the laser displacement sensor before (a) and after (b) correction.

Obviously, to improve the accuracy of determining the localization of heterogeneity in the phantom of the human chest, it is necessary to perform an adjustment of the coordinates of the sensor, which can be carried out at post-processing of measurement results on a PC. One of the simplest and most obvious correction methods is zeroing of the coordinates of the extreme points and shifting the coordinates of the other points by the corresponding value.

From figure $4, \mathrm{~b}$ it can be seen that after the adjustment, the trajectory of the laser displacement sensor in the scanning process looks more evenly. However, in order to speak about the legality of making an adjustment, it is also necessary to analyze its influence on the measurement results. Figure 5 shows the results of a radio frequency scanning of a human chest phantom $[10,11]$ with an introduced heterogeneity - a metal disk $10 \mathrm{~cm}$ in diameter before (a) and after (b) performing the trajectory correction. The frequency of the probing microwave signal is $1150 \mathrm{MHz}$.

From Figure 5 it can be seen that the adjustment of the trajectory of the laser displacement sensor does not distort the shape or size of the heterogeneity (metal disc). Thus, the application of this adjustment is permissible.

Analysis of the results allows to conclude about the error of measuring the size of heterogeneity about $15 \mathrm{~mm}$. To improve the accuracy of determining the size of heterogeneity, it is necessary to use an antenna applicator with a narrower radiation pattern, which will lead to a proportional increase in its size. In this connection, a compromise is needed between the dimensions of the antenna and the accuracy of the results. A significant increase in the frequency of the probing signal is not applicable, since the greatest contrast of the results of radio frequency scanning takes place in a limited frequency band of the probing microwave signals [12].

Thus, despite the fact that the accuracy of determining the coordinates when using a laser displacement sensor is lower than in the case of an automatic positioning system based on stepper motors [7], the developed positioning system can be used to determine the coordinates of the radiating module in the process of radio frequency scanning to diagnose broncho-pulmonary diseases after performing the described correction procedure during the post-processing of measurement results. 

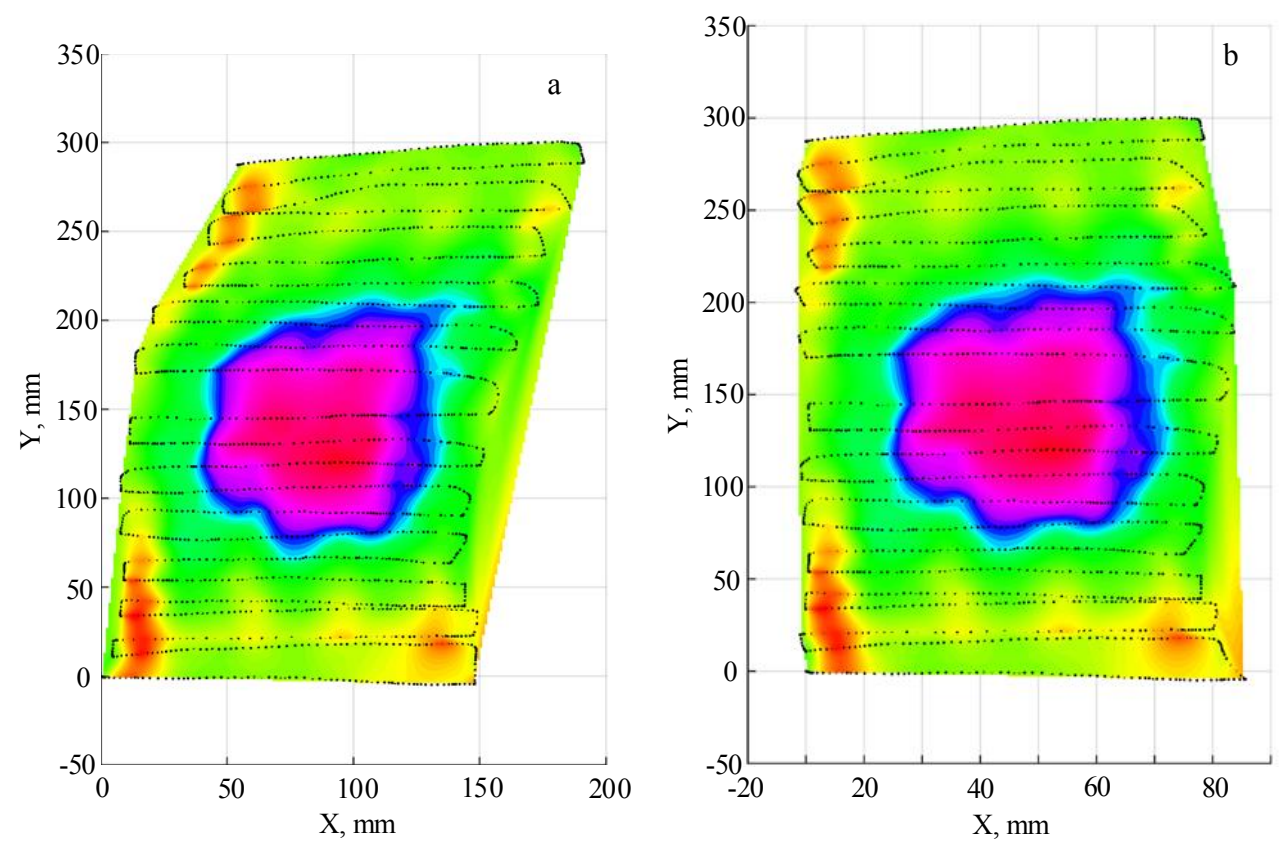

Fig. 5. The results of the measurement of the transmission coefficient before (a) and after (b) correction.

Figures 6 and 7 shows the results of scanning a thorax phantom with the inhomogeneity in the form of a hollow ball made of flexible radio transparent material filled with $100 \mathrm{ml}$ of $0.9 \% \mathrm{NaCl}$ solution by the probing microwave signal with a frequency of $1150 \mathrm{MHz}$.

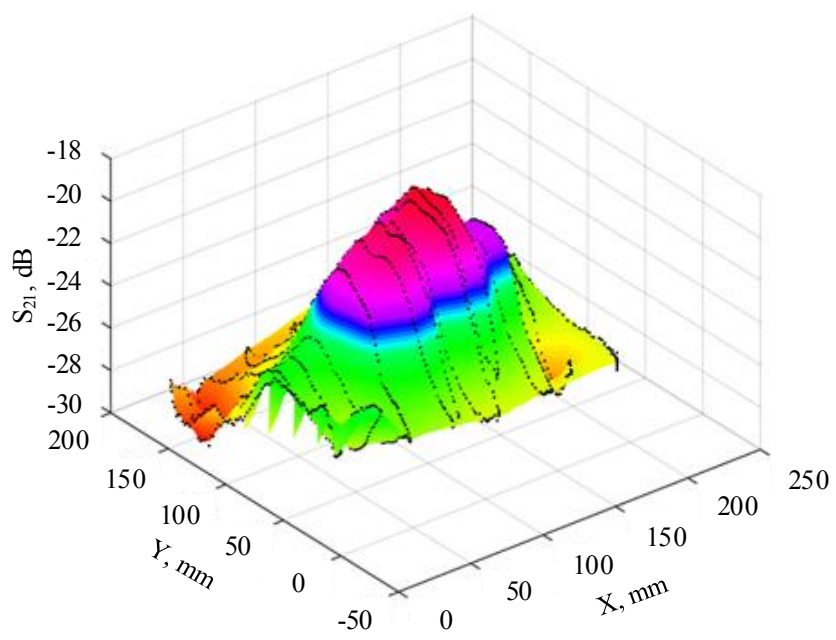

Fig. 6. Results of radio frequency scanning in 3D format.

This heterogeneity is an imitation of the presence in the patient's chest of an excessive amount of sputum, which is a consequence of the disease. In Figure 6, the results of radio frequency scanning are presented in $3 \mathrm{D}$, with the values of the coefficient of transmission of the probing microwave signal through the phantom of the chest with heterogeneity along the vertical axis. Figure 7 shows a two-dimensional projection of the results obtained on the XY plane. 


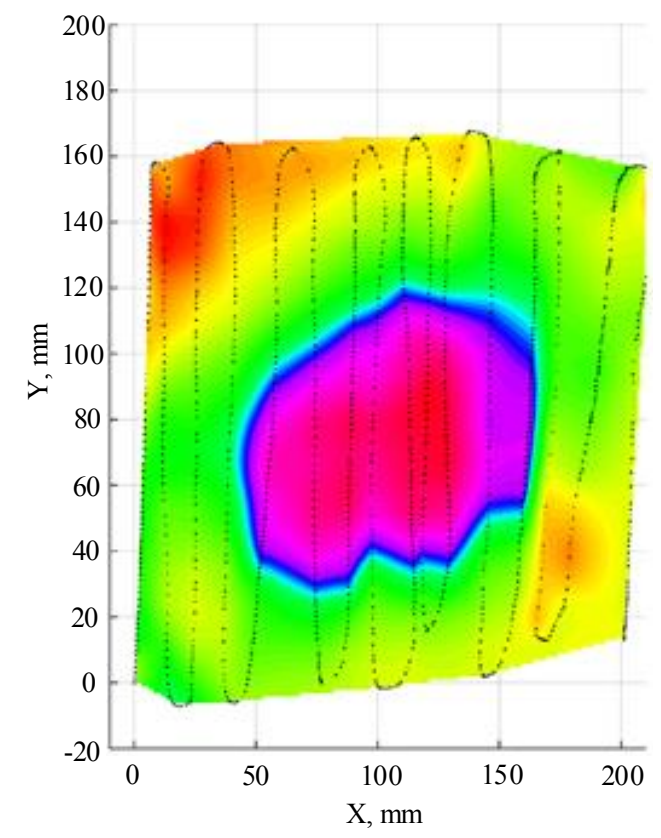

Fig. 7. Results of radio frequency scanning in $2 \mathrm{D}$ format.

Analysis of the results suggests that the proposed method and the developed system of radiofrequency scanning allows determining the presence in the human chest of both heterogeneities of a sharp nature - metal objects (Figure 5) and heterogeneities resulting from bronchopulmonary diseases - excessive amount of sputum (Figures 6 and 7). The difference between the values of the transmission coefficient of the probing microwave signal in this case is $5-15 \mathrm{~dB}$, which is sufficient for detecting inhomogeneities. It is difficult to accurate determining the boundaries of heterogeneity in Figs. 6 and 7, since the inhomogeneity did not have a rigid form and was deformed when it was inserted into the phantom of the chest.

\section{Conclusion}

Thus, this paper presents the structure of a semi-automatic system of non-invasive harmless diagnosis of bronchial asthma and other bronchopulmonary diseases. The components of the system are considered and it is shown that it is possible to design a diagnostic system in a portable compact design by using a laser displacement sensor instead of a bulky positioning system. The advantages of the proposed system are the simplicity of the implementation of the diagnostic system and examination, harmlessness, non-invasiveness of the examination procedure, no need to perform breathing maneuvers. These advantages allow us to consider the proposed system as an addition to the existing methods of diagnosing bronchial asthma, as well as an express method for diagnosing bronchopulmonary diseases or a system for periodically monitoring the health of a patient undergoing inpatient treatment or not having permanent access to a large medical center equipped with expensive equipment.

The paper is based on the research results, which was conducted in the framework of project MK-5780.2018.8 with the support of the Grant Council of the President of the Russian Federation. 


\section{References}

1. R. Yadav, D. Gumber, Bronchial asthma: an unmet disease, Int J Pharm Sci Res, v. 8(4), pp. 1514-1521 DOI: 10.13040/IJPSR.0975-8232.8(4).1514-21 (2017)

2. A. Poreva, V. Vaityshyn, V. Timofeyev, A. Honcharenko, Improving of lung sounds registration device for further signal processing, Proceedings of 2017 IEEE 37th International Conference on Electronics and Nanotechnology (ELNANO) DOI: 10.1109/ELNANO.2017.7939773 (18-20 April 2017)

3. R. B. Salah, T. Hadidi, S. Chabchoub, Intelligent diagnosis method of cardiovascular anomalies using medical signal processing, Proceedings of 2015 World Congress on Information Technology and Computer Applications (WCITCA), DOI: 10.1109/WCITCA.2015.7367032 (11-13 June 2015)

4. M. R. Neuman, G. D. Baura, S. Meldrum, O. Soykan, M. E. Valentinuzzi, R. S. Leder, S. Micera, Y.-T. Zhang, Advances in medical devices and medical electronics, Proceedings of IEEE, v. 100, pp. 1537-1550 DOI: 10.1109/JPROC.2012.2190684 (2012)

5. Z. Cao, R. Zhu, R.-Y. Que, A wireless portable system with microsensors for monitoring respiratory diseases, IEEE Transactions on biomedical engineering, v. 59, No. 11. DOI: 10.1109/TBME.2012.2211354 (2012)

6. S. Nousias, A.S. Lalos, G. Arvanitis, K. Moustakas, T. Tsirelis, D. Kikidis, K. Votis, D. Tzovaras, An mHealth system for monitoring medication adherence in obstructive respiratory diseases using content based audio classification, IEEE Access, v. 6, pp. 11871-11882, DOI: 10.1109/ACCESS.2018.2809611 (2018)

7. M. Vitacca, A. Montini, L. Comini, How will telemedicine change clinical practice in chronic obstructive pulmonary disease?, Therapeutic Advances in Respiratory Disease, v. 12, pp. 1-19. DOI: $10.1177 / 1753465818754778$ (2018)

I. V. Semernik, O. E. Semernik, A. V. Dem'yanenko, A. A. Lebedenko, A Method for Noninvasive Diagnosis of Bronchial Asthma Based on Microwave Technology. Biomedical Engineering, v. 51(2), pp. 124-127 DOI: 10.1007/s10527-017-9698-3 (2017)

8. I. V. Semernik, A. V. Dem'yanenko, F. S. Topalov, Y. V. Nevstruev, Prospects for designing a portable system for monitoring of the patient's condition with bronchial asthma, 2019 IEEE Conference of Russian Young Researchers in Electrical and Electronic Engineering Proceedings, St. Petersburg, Russia, DOI: 10.1109/EIConRus. 2019.8657090 (January 28-31, 2019)

9. I. V. Semernik, A. V. Dem'yanenko, F. S. Topalov, Y. V. Nevstruev, Automatic system for early diagnosis of pathological changes in the bronchopulmonary system based on radiofrequency scanning of the chest, 2019 IEEE Conference of Russian Young Researchers in Electrical and Electronic Engineering Proceedings, St. Petersburg, Russia, DOI: 10.1109/EIConRus.2019.8657188 (January 28-31, 2019)

10. I. V. Semernik, O. E. Semernik, A. V. Dem'yanenko, A. A. Lebedenko, F. S. Topalov. Device for non-invasive diagnosis of bronchopulmonary diseases based on microwave technologies, 2018 IEEE Conference of Russian Young Researchers in Electrical and Electronic Engineering Proceedings, St. Petersburg, Russia DOI: 10.1109/EIConRus. 2018.8317312 (January 29 - February 01, 2018)

11. A. V. Dem'yanenko, I. V. Semernik, Y. V. Nevstruev, Designing of broadband microwave applicator for the bronchial asthma diagnosis device, 2018 IEEE Conference of Russian Young Researchers in Electrical and Electronic Engineering Proceedings, St. Petersburg, Russia, DOI: 10.1109/EIConRus.2018.8317306 (January 29 - February 01, 2018) 
12. O. E. Semernik, I. V. Semernik, A. V. Dem'yanenko, A. A. Lebedenko, Y. V. Nevstruev, Designing of the human chest phantom, Proceedings of the 26th International Conference "Microwave \& Telecommunication Technology" (CriMiCo'2016), Sevastopol, Russia, 1818-1824. (September 4-10, 2016)

13. I. V. Semernik, A. V. Dem'yanenko, F. S. Topalov, Ch. V. Samonova, O. E. Semernik, A. A. Lebedenko, Bronchial Asthma Diagnosis Device Based on Microwave Tecnologies, Proceedings of 2017 Radiation and Scattering of Electromagnetic Waves RSEMW-2017, Divnomorskoe, Russia. DOI: 10.1109/RSEMW.2017.8103648 (June 26-30, 2017) 